OPEN ACCESS

Edited by:

Qixiao Zhai,

Jiangnan University, China

Reviewed by:

Guanxiang Liang,

University of Pennsylvania,

United States

Ya-Ting Wang,

Washington University in St. Louis,

United States

${ }^{*}$ Correspondence:

Ling Yaqin

lingyq@/zu.edu.cn

Pei Lingpeng

Ippei@hotmail.com

Specialty section:

This article was submitted to Microbiome in Health and Disease,

a section of the journal

Frontiers in Cellular and

Infection Microbiology

Received: 18 July 2020 Accepted: 02 December 2020 Published: 14 January 2021

Citation:

Enqi W, Jingzhu S, Lingpeng $P$ and Yaqin $L$ (2021) Comparison of the Gut Microbiota Disturbance in Rat Models of Irritable Bowel Syndrome Induced by Maternal Separation and Multiple Early-Life Adversity. Front. Cell. Infect. Microbiol. 10:581974.

doi: 10.3389/fcimb.2020.581974

\section{Comparison of the Gut Microbiota Disturbance in Rat Models of Irritable Bowel Syndrome Induced by Maternal Separation and Multiple Early-Life Adversity}

\author{
Wu Enqi, Song Jingzhu, Pei Lingpeng ${ }^{*}$ and Ling Yaqin * \\ Key Laboratory of Ethnomedicine (Minzu University of China), Ministry of Education, Beijing, China
}

Background: The study aimed to identify the effects of modeling procedures on bacterial communities and to investigate whether different modeling procedures lead to consistent patterns of gut microbiome compositions.

Methods: Two irritable bowel syndrome (IBS) rat models maternal separation (MS) alone and multiple-early-adversity modeling (MAM) were established and the gut microbiome were analyzed using 16S-rRNA-based high-throughput sequencing methods.

Results: Rats from both models exhibited visceral hypersensitivity and the two model groups exhibited differences in the extent of visceral sensitivity and fecal water content. The microbial community structure of the two models exhibited significant differences compared to the controls, while the two model groups also exhibited significant differences between them. Furthermore, microbial community functional predictions suggested that the two models exhibited different abundances of metabolisms and pathways. Several common and distinct characteristic differences were also observed between the two model groups. Alloprevotella were more abundant in both model groups, while Butyricicoccus, Turicibacter, Ruminococcus, and Clostridium_sensu_stricto along with the family it belongs to were less abundant relative to controls. In addition, the abundance of Clostridium_IV, Corynebacterium, Rothia, Elusimicrobium, Romboutsia, Allobaculum, Parasutterella, and their related taxa were specifically associated with MS group, whereas Butyricimonas and Vampirovibrio along with its related taxa were specifically associated with MAM group. Among those, Butyricimonas, Butyricicoccus and Corynebacterium were found to partially mediate early adversity exposure-induced visceral hypersensitivity.

Conclusions: Our results highlight the importance in evaluating gut microbiota characteristics in IBS research while also systematically considering potential modeling procedural differences. The microbial compositional/functional differences identified in this study were suggestive to further investigation of mechanisms of early adversity induced IBS.

Keywords: microbiota, irritable bowel syndrome, rat, maternal separation, multiple early-life adversity, visceral hypersensitivity 


\section{INTRODUCTION}

Irritable bowel syndrome (IBS) is a chronic functional gastrointestinal disorder characterized by abdominal discomfort, bloating, and disturbed defecation in the absence of any identifiable abnormalities indicative of organic gastrointestinal disease (Holtmann et al., 2016). The pathophysiology of IBS is likely heterogeneous and may involve visceral hypersensitivity, gut barrier dysfunction, altered brain-gut signaling, immune dysregulation, altered microbiota characteristics, and psychosocial factors (Halland and Saito, 2015; Ford et al., 2017).

Various animal models have been developed to study the underlying mechanisms of IBS, among which the rodent maternal separation (MS) model is well-established and based on the premise that environmental changes during the early postnatal period can have long-lasting effects into adulthood, including visceral hyperalgesia and altered colonic motility (Fukui et al., 2018; Rincel and Darnaudery, 2019). Combined animal models have the advantage of allowing the mimicking of multiple etiological factors of IBS and are developed by exposing animals to multiple stimuli, as has been used in several IBS studies (Gong et al., 2014; Zhuang et al., 2016; Li et al., 2019).

Increasing data have demonstrated the relationship between alterations in gut microbiota characteristics and IBS. It was reported that IBS patients harbor altered gut microbiota compared to healthy controls, while fecal microbiota transplantation from healthy donors to IBS patients has shown promise in alleviating IBS symptoms (Tap et al., 2017; Aroniadis et al., 2019). Among animal model studies, rats exposed to early-life adversity, including maternal separation, intestinal inflammation, or infection, restraint stress, and water avoidance stress have been shown to display visceral hypersensitivity and altered microbiota profiles in adulthood (Ma et al., 2019; Rincel et al., 2019; Zhang et al., 2019). However, an understanding of the specific impacts of different modeling methodologies on gut microbiota is currently lacking, and the microbial signatures that are consistently associated with certain pathophysiological changes in IBS have not been identified.

In the present study, two common IBS rat models (MS alone and multiple early adversity modeling) were established. Highthroughput microbial community analysis of their colon contents was then performed to identify the influence of modeling procedures on bacterial community and investigate whether different modeling procedures lead to consistent patterns of gut microbiota compositions. In addition, the possible mediating effects of gut microbial composition were explored between early adversity and altered pathophysiological variants.

\footnotetext{
Abbreviations: AWR, Abdominal withdrawal reflex; ACME, Average causal mediation effect; CTRL, Control; CRD, Colorectal distension; ER, Expected error; IBS, Irritable bowel syndrome; KEGG, Kyoto Encyclopedia of Genes and Genomes; KO, KEGG orthologs; LEfSe, Linear discriminant analysis Effect Size; MS, Maternal separation; MAM, Multiple early adversity model; ANOVA, Oneway analysis of variance; PCoA, Principal coordinates analysis; PND, Postnatal day; SPF, specific pathogen free; SD, Standard deviations; TE, Total effect; ZOTUs, Zero-radius operational taxonomic units.
}

\section{MATERIALS AND METHODS}

\section{Animals and Ethics Statement}

Pregnant specific pathogen free (SPF) Sprague Dawley rats were obtained from SPF Biotechnology Co., Ltd. (Beijing, China), and arrived in the lab on day 15 of their pregnancies. Rats were housed in standard polypropylene shoebox cages $(42 \times 20.5 \times$ $20 \mathrm{~cm}$ ) on hardwood chip bedding in a designated room with controlled lighting $\left(12: 12 \mathrm{~h}\right.$ light-dark cycle) at $22^{\circ} \mathrm{C}$ to $24^{\circ} \mathrm{C}$ and allowed free access to water and a standard diet. Pregnant rats were checked daily for litter, and the day of birth was defined as the postnatal day (PND) 0 for the litter. After delivery, the dams and litters were housed together until weaning (PND22). After weaning on PND22, male and female rats were separated, and three animals were kept in a single standard cage. Only male rats were included in this study to avoid the effects of hormones ( $\mathrm{n}=$ 7 for each group). The experimental animal protocol was approved by the Ethics Committee of Minzu University of China (ECMUC2019005AO). The study was carried out in a Good Laboratory Practice (GLP) accredited laboratory.

\section{Study Design}

Pregnant rats were randomly divided into three groups: the control (CTRL), MS, and multiple early adversity model (MAM) groups. Rats were left undisturbed in the CTRL group except for routine cage cleaning every 2 days. In the MS group, the maternal separation was performed once daily during PND1-PND21. Separations were conducted between 9:00 AM and 12:00 noon by placing the offspring in plastic cages placed on the top of heating pads set at $30^{\circ} \mathrm{C}$ to $33^{\circ} \mathrm{C}$ in a separate room from the main holding room. In the MAM group, the maternal separation was performed once daily during PND1-PND21, acetic acid instillation was performed once daily during PND15-PND28, and the rats were restrained for $3 \mathrm{~h}$ every day during PND29-PND42. Rats are typically reared by PND43. Rats were anesthetized for acetic acid instillation, and $0.5 \%$ acetic acid was slowly instilled into the colon with a plastic tube (external diameter of $1 \mathrm{~mm}$ ) at a depth of $6 \mathrm{~cm}$ from the anus and then maintained for $3 \mathrm{~min}$. The acetic acid dose on the first day was $0.2 \mathrm{ml}$ and was increased by $0.1 \mathrm{ml}$ every day. After increasing to $0.5 \mathrm{ml}$, enemas were administered at a dosage of $0.5 \mathrm{ml}$ every day. To model restraint stress, rats were placed in a transparent plastic restraint cylinder, in which they could move forward and backward but could not turn around for $3 \mathrm{~h}$. All rats were weighed once a week after PND22. Abdominal withdrawal reflex (AWR) scores in response to colorectal distension were measured at PND78, and fecal water content was measured at PND82 followed by recovery of distal colon contents at PND84. All of the rats were anesthetized using Isoflurane and were sacrificed by exsanguinations after blood collected from the posterior vena cava on PND84 as described in previously (Parasuraman et al., 2010).

\section{Visceral Sensitivity Assessment}

Visceral hypersensitivity was evaluated using the AWR score at PND78, as described previously (Xu et al., 2009; Wang et al., 2018). Briefly, a flexible balloon $(6 \mathrm{~cm})$ constructed from a 
surgical glove finger was attached to Tygon tubing and inserted $8 \mathrm{~cm}$ into the descending colon via the anus and held in place by taping the tubing to the tail. Rats were placed in transparent plastic cubicles $(8 \times 10 \times 20 \mathrm{~cm})$ and allowed to adapt for $10 \mathrm{~min}$. colorectal distension was then established by rapidly inflating the balloon to constant pressure. Pressure was measured using a sphygmomanometer connected to a pressure transducer. The balloon was inflated to various pressures $(20,30,40,50,60,70$, 80,90 , and $100 \mathrm{~mm} \mathrm{Hg}$ ) for a 10 -s stimulation period followed by a 1-min rest period. Behavioral responses to colorectal distension were measured by visual AWR observations by two blinded observers, and AWR score assignments were as follows: 0, normal behavior without response; 1, slight head movement without abdominal muscle contraction; 2, contraction of abdominal muscles; 3, lifting of abdominal wall; and 4, body arching and lifting of pelvic structures. The minimal distention pressures that evoked abdominal visceromotor responses corresponding to AWR scores of 2, 3, and 4 were recorded by both observers and defined as the distension threshold.

\section{Fecal Water Content}

Fresh feces expelled by each rat were collected on PND82 in $5 \mathrm{ml}$ sterile tubes and immediately weighed, followed by drying them in an oven to calculate fecal water content with the following formula: water content $\%=$ [wet weight of the feces $(\mathrm{g})-$ dried weight of the feces $(\mathrm{g})] /$ wet weight of the feces $(\mathrm{g}) \times 100 \%$.

\section{Collection of the Colorectal Fecal Contents}

Colorectal fecal contents were collected just after sacrifice. Fresh fecal pellets were collected directly from the distal colon, placed in 5-ml sterile tubes, and preserved with $4 \mathrm{M}$ guanidine thiocyanate solution at $-20^{\circ} \mathrm{C}$, then transferred to the laboratory within $24 \mathrm{~h}$ and stored at $-80^{\circ} \mathrm{C}$ prior to DNA extraction and characterization of the microbiota using $16 \mathrm{~S}$ rRNA gene sequencing.

\section{Histological Examination of Colonic Inflammation}

The histological tissues were fixed in formalin for $12 \mathrm{~h}$, and pathological sections were generated at a thickness of $5 \mu \mathrm{m}$ and processed for hematoxylin-eosin staining. The slides were observed under an optical microscope, and inflammatory cells were observed by a pathologist blinded to the treatments.

\section{Microbiota Sequencing}

Total bacterial DNA was extracted from samples using the Power Soil DNA Isolation Kit (MO BIO Laboratories) according to the manufacturer's protocol. DNA quality and quantity were assessed by the ratios of $260 \mathrm{~nm} / 280 \mathrm{~nm}$ and $260 \mathrm{~nm} / 230 \mathrm{~nm}$. Then DNA was stored at $-80^{\circ} \mathrm{C}$ until further processing.

The V3-V4 region of the bacterial 16S rRNA gene was amplified with the common primer pair (Forward primer, 5' ACTCCTACGGGAGGCAGCA-3'; reverse primer, 5'- GGA CTACHVGGGTWTCTAAT-3') combined with adapter sequences and barcode sequences. PCR amplification was performed in a total volume of $50 \mu \mathrm{l}$, which contained $10 \mu \mathrm{l}$ buffer, $0.2 \mu \mathrm{l}$ Q5 High-Fidelity DNA Polymerase, $10 \mu \mathrm{l} \mathrm{High} \mathrm{GC}$ Enhancer, $1 \mu \mathrm{ldNTP}, 10 \mu \mathrm{M}$ of each primer and $60 \mathrm{ng}$ genome DNA. Thermal cycling conditions were as follows: an initial denaturation at $95^{\circ} \mathrm{C}$ for $5 \mathrm{~min}$, followed by 15 cycles at $95^{\circ} \mathrm{C}$ for $1 \mathrm{~min}, 50^{\circ} \mathrm{C}$ for $1 \mathrm{~min}$, and $72^{\circ} \mathrm{C}$ for $1 \mathrm{~min}$, with a final extension at $72^{\circ} \mathrm{C}$ for $7 \mathrm{~min}$. The PCR products from the first step PCR were purified through VAHTSTM DNA Clean Beads. A second round PCR was then performed in a $40-\mu$ l reaction which contained $20 \mu \mathrm{l} 2 \times \mathrm{Ph} \mu$ sion HF MM, $8 \mu \mathrm{l} \mathrm{ddH}_{2} \mathrm{O}, 10 \mu \mathrm{M}$ of each primer, and $10 \mu \mathrm{l}$ PCR products from the first step. Thermal cycling conditions were as follows: an initial denaturation at $98^{\circ} \mathrm{C}$ for $30 \mathrm{~s}$, followed by 10 cycles at $98^{\circ} \mathrm{C}$ for $10 \mathrm{~s}, 65^{\circ} \mathrm{C}$ for $30 \mathrm{~s} \mathrm{~min}$, and $72^{\circ} \mathrm{C}$ for $30 \mathrm{~s}$, with a final extension at $72^{\circ} \mathrm{C}$ for $5 \mathrm{~min}$. Finally, all PCR products were quantified by Quant-iT ${ }^{\mathrm{TM}}$ dsDNA HS Reagent and pooled together. High-throughput sequencing analysis of bacterial rRNA genes was performed on the purified, pooled sample using the Illumina Hiseq 2500 platform $(2 \times 250$ paired ends) at Biomarker Technologies Corporation, Beijing, China.

\section{Bioinformatic Analyses}

Raw 16S rRNA gene sequence reads were demultiplexed and then trimmed, merged, and filtered using the Usearch v11.0.667linux32 program using the UNOISE pipeline. Pairedend sequence reads with an expected error $(E R)$ value $>1.00$ were removed during filtering. Sequences were dereplicated and classified into zero-radius operational taxonomic units (ZOTUs) after denoising (error-correcting) and chimera checking. The RDP classifier was then used to assign taxonomic classifications to $16 \mathrm{~S}$ rRNA gene sequences with a confidence threshold of $80 \%$. Representative sequences from the ZOTUs were aligned and further filtered to create a phylogenetic tree using the QIIME pipeline. The ZOTU table was subsampled randomly to the number of reads in the smallest sample $(19,511)$ in order to obtain equal sequencing depths among samples. The functional profiles of the microbial communities were also predicted using the open-source $\mathrm{R}$ package Tax4Fun and the Kyoto Encyclopedia of Genes and Genomes (KEGG) ortholog dataset based on $16 \mathrm{~S}$ rRNA gene profiles.

\section{Statistical Analyses}

Categorical variables were evaluated as frequencies and percentages. Chi-squared tests and Fisher's exact tests were then used to assess statistical associations between variables. Numerical variables were expressed as means \pm standard deviations (SD). One-way analysis of variance (ANOVA) tests were used to compare differences in measured parameters among groups. Wilcoxon rank-sum test or Kruskal-Walls test were used to compare differences of nonparametric (not normally distributed) data

The Chao1, observed OTU numbers (obs_otus), phylogenetic diversity (PD_whole_tree), Shannon and Simpson indices were used to evaluate community richness and diversity of the gut microbial communities. Principal coordinates analysis (PCoA), adonis tests, and db-RDA analyses were used to evaluate differences among communities based on beta diversity metrics 
including the unweighted and weighted Unifrac distance metrics. Finally, the Linear discriminant analysis Effect Size (LEfSe) analysis with a threshold of $+/-2$ were used to explore significant differences among treatment groups using the relative abundance data of taxa and the KEGG-based ortholog and pathway abundances.

\section{Mediation Analyses}

To further assess the contribution of alteration in gut microbiota to the association between early adversity and the altered physiological factors in adulthood including body weight, visceral hypersensitivity, and fecal water content, mediation analyses were conducted using the "mediation" package in R. Bacterial taxa were considered as mediators in mediation analysis to evaluate the apparent causal effects of early adversity on the physiological factors in adulthood. The total effect (TE) and average causal mediation effect (ACME) values were determined between predictors and outcomes via the mediators, and the percent of mediation effect was estimated using a two-tailed $P$-value cutoff of $<0.05$ to assess statistical significance.

\section{RESULTS}

\section{Model Assessment}

Visceral sensitivity is one of the most important pathophysiological characteristics of IBS. Here, we used colonic stretch stimulation
AWR scores to evaluate the visceral sensitivity of treatment groups. Both MS and MAM groups exhibited increases in visceral hypersensitivity to colorectal distension compared to control group individuals (Figures 1A, B). The MAM group showed even higher visceral hypersensitivity than did the MS group. The AWR scores were significantly higher in the MAM rats compared to those of the control group at pressures of 40,50,60, 70, and 80 $\mathrm{mmHg}$ and in MS rats at pressures of 50 and $60 \mathrm{mmHg}$. The threshold values for the MS group for AWR score 2 were significantly lower than those of the control group, while the threshold values of AWR scores 2, 3, and 4 were all significantly lower in the MAM group compared to the control group. These results thus indicate that visceral hypersensitivity was induced in both the MAM and MS groups.

No statistically significant differences were observed in body weights between different model groups, although the body weights of rats in the MS group were generally higher than those of the other two groups (Figure 1C). Fecal water content was measured to evaluate differences in general stool features, and a significant decrease in the fecal water content of MAM rats was observed compared to control rats $(11.6 \% \pm 4.3 \%$ vs $20.1 \% \pm 4.7 \%, \mathrm{p}=0.0059, \mathrm{n}=7)$. No such effect was observed between the control and MS rats $(18.3 \% \pm 4.8 \%)$ (Figure 1D). Histological examination did not indicate remarkable inflammatory features in all groups on PND84, suggested that the two modeling methods did not cause obvious intestinal organic lesions.
A
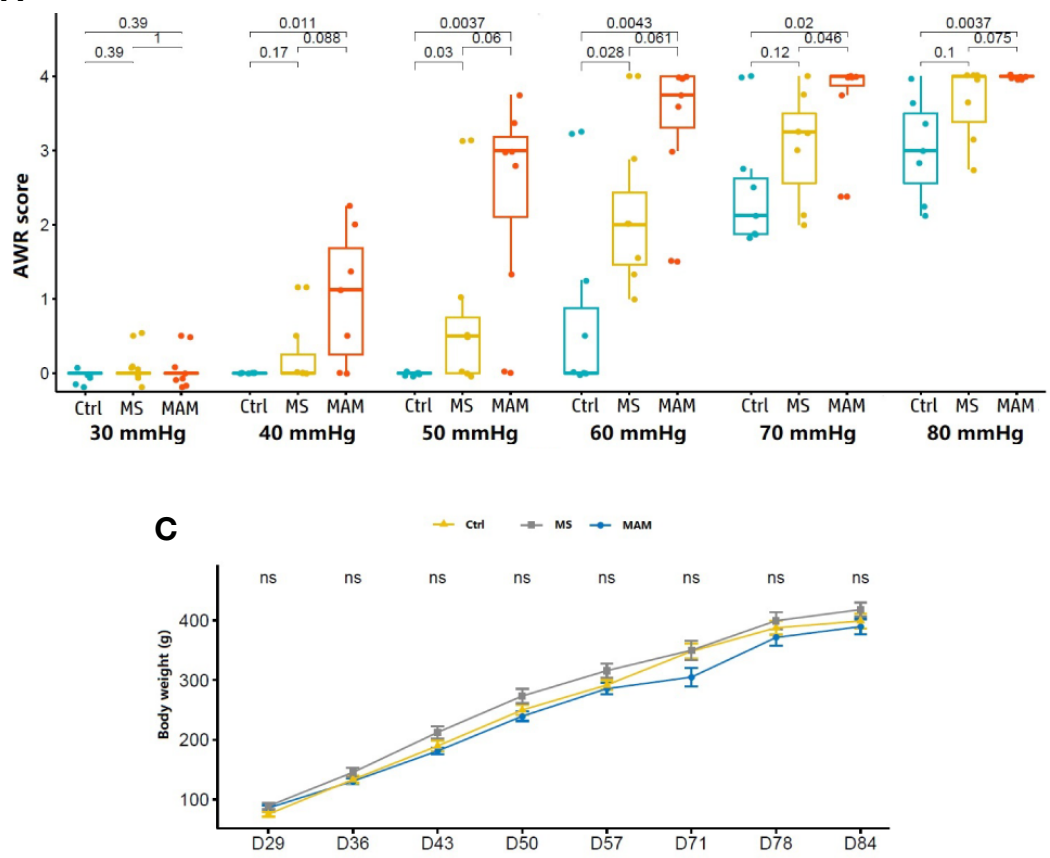

B
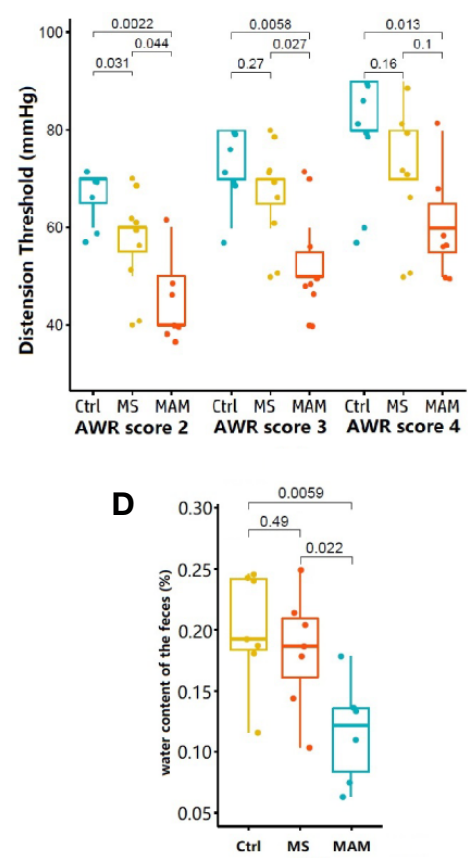

FIGURE 1 | Comparison of visceral hypersensitivity, body weight, and fecal water content in rats of different model groups ( $\mathrm{n}=7$ for each group). (A) Abdominal withdrawal reaction (AWR) scores in response to graded colorectal distension (CRD). (B) Distension threshold values for AWR scores of 2, 3, and 4. (C) Body weight variation of rats (ns, not significant). (D) Fecal water content for rats in the control, MS, and MAM groups. 


\section{Overall Assessment of Intestinal Microbiota Variation}

A total of 1,395,522 paired-end 16S rRNA gene sequence reads covering the V3-V4 hypervariable regions were generated from 21 samples, with an average of $66,453( \pm 13,675.83 \mathrm{SD})$ reads per sample, and ranging from 45,296 to 80,200 per sample. After trimming and filtering, a total of 1,058,795 high-quality pairedend reads remained. Further denoising resulted in the removal of 1.091 chimeric sequences.

A total of 4,498 zero-radius operational taxonomic units (ZOTUs) were ultimately generated and further used for data analysis. All ZOTUs were annotated by comparison against the RDP database. Among these, 4,453 ZOTUs were annotated at the phylum levels, comprising 7 phyla. In addition, a total of 4,401 , $4,335,4,125$ and 1,940 ZOTUs were annotated at the class, order, family, and genus level comprising 13 classes, 14 orders, 21 families, and 38 genera, respectively (Figure 2).

\section{Comparison of Microbial Community Structures Among Different Model Groups}

Several different richness and diversity indices were calculated for the microbial communities of different treatment groups after randomly subsampling the ZOTU table to 18,429 reads per sample, which was the size of the smallest sample. No significant differences were observed for all five diversity indices among the different model groups (Figure 3). To examine bacterial community structural differences among model groups, two distance matrices were created from the subsampled ZOTU table based on the unweighted Unifrac distance metric and the weighted Unifrac distance metric. Principal coordinate analysis (PCoA) of beta-diversity differences based on the unweighted UniFrac distance metric indicated that the microbial communities from the three model groups were completely differentiated into three different clusters. Furthermore, PCoA based on the weighted Unifrac distance metric also indicated the partial separation of the microbiota communities based on the different model groups (Figures 4A, B). Adonis tests and db-RDA analyses of both distance matrices indicated the presence of significant associations between the different model groups and bacterial community structures.

Comparison of intra-group and inter-group distances for each model group based on the unweighted Unifrac distance metric suggested that the extent of variation within the MS and MAM groups was significantly higher than that of the control group, with no significant difference between the two model groups, indicating that the modeling process not only altered the structure of the gut microbiota, but the extent of instability of the gut microbiota also increased significantly. Pairwise comparisons of the distances between the groups indicated that the distance between the two model groups was significantly higher than the distances between either the MS or MAM communities to those of the control group, suggesting that the gut microbiota structure varies in different directions based on the modeling methodology (Figure 4C).

The comparison of the intra-group and inter-group distances of each model group based on the weighted Unifrac distance metric did not yield statistically significant differences (Figure 4D).

\section{Differences in Microbiota Composition and Functionalities Among Control and Maternal Separation Groups}

A total of 26 taxa exhibited significantly different abundances in the comparison between the control and MS groups (Figure 5A). Corynebacterium and the family to which it belongs as well as Alloprevotella were more abundant in the MS group. The genus Rothia and the family to which it belongs along with Elusimicrobium and the family, order, class, and phylum to which it belongs were more abundant in the control group. Other taxa that were more abundant in the control group communities included Clostridium sensu stricto and the family to which it belongs, Ruminococcus, Romboutsia and the family to which it belongs, Butyricicoccus, Clostridium IV, Allobaculum and the family, order, and class to which it belongs, Turicibacter, and lastly, Parasutterella and the family, order, and class to which it belongs.

In addition, $10 \mathrm{KOs}$ and 8 pathways had significantly different inferred abundances between the control and MS groups (Figures 5C, E). Specifically, we observed higher abundances of the pathways in the MS group compared to the control group involving the citrate (TCA) cycle (ko00020), protein export (ko03060), carbohydrate digestion and absorption (ko04973), and NF-kappa B signaling (ko04064), along with lower abundances of alanine, aspartate, and glutamate metabolism (ko00250), non-ribosomal peptide structures (ko01054), tyrosine metabolism (ko00350), and porphyrin and chlorophyll metabolism (ko00860).

\section{Differences in Microbiota Composition and Functionalities Among Control and Multiple Early Adversity Model Groups}

Comparison of the control and MAM groups indicated the presence of 14 taxa with significant differences (Figure 5B). Specifically, the genus Vampirovibrio and the family, order, class, and phylum to which it belongs as well as Alloprevotella were more abundant in the MAM group. In contrast, Clostridium sensu stricto and the family, order, and class to which it belongs along with the Butyricimonas, Turicibacter, Ruminococcus, and Butyricicoccus genera were more abundant in the control group samples.

LEfSe analysis indicated that $8 \mathrm{KOs}$ exhibited significant differences comparing the control and MAM groups (Figure 5D). At the functional pathway level, 8 pathways had significantly different inferred abundances among the control and MAM groups (Figure 5F). In the MAM group, pathways that were significantly more abundant than in the control group included epithelial cell signaling in Helicobacter pylori infection (ko05120), galactose metabolism (ko00052), glutathione metabolism (ko004800), and the Fanconi anemia pathway (ko03460), while significantly lower pathways included morphine addiction (ko05032), thiamine metabolism 
A

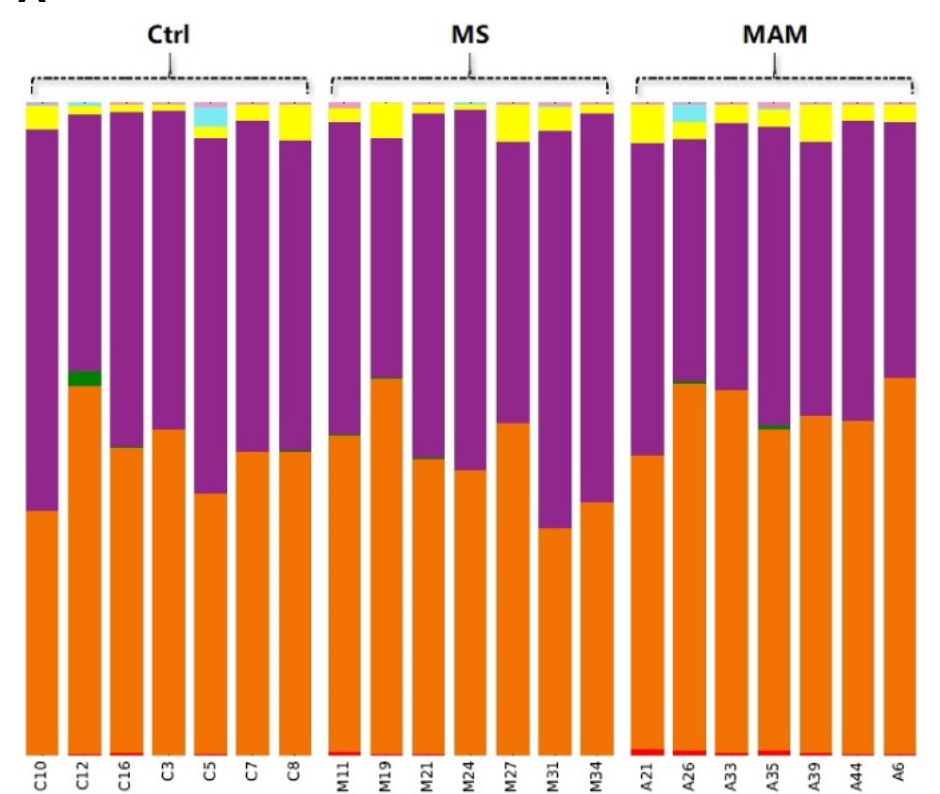

B

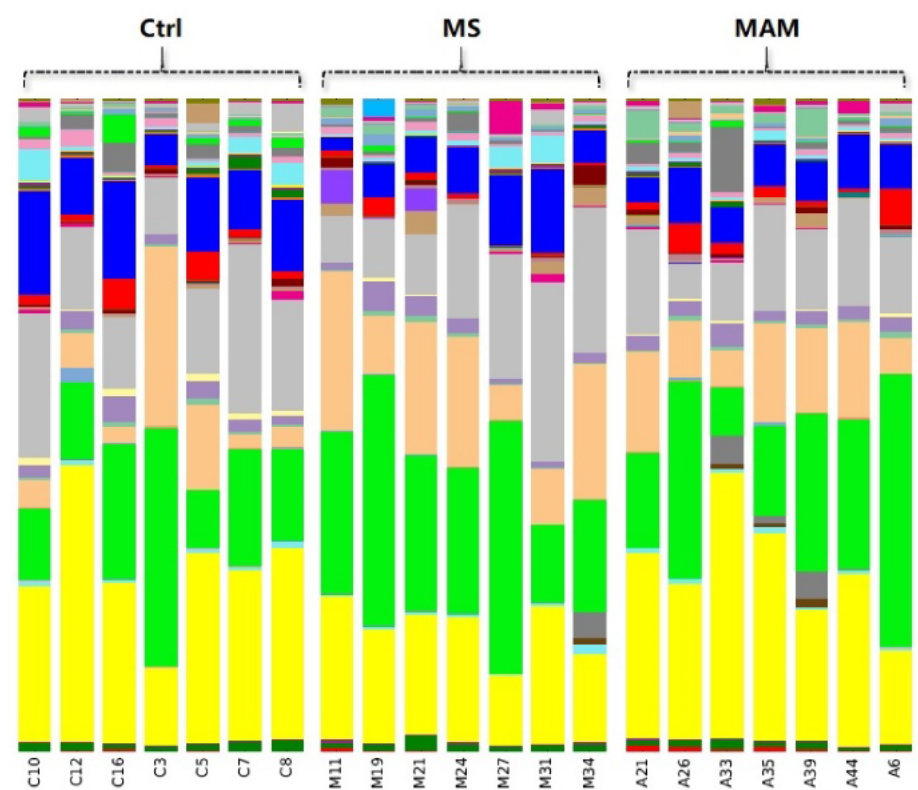

n_Actinobacteria

p_Bacteroidetes

D_Elusimicrobi

p Proteobacteri

p_Tenericutes

p_Verrucomicrobia

D_other

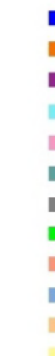

\section{三.end}

- g_Corynebacterium

g_Rothia

g Bacteroides

g_Barnesiella

- g_Butyricimonas

g Parabacteroides

- g_Alloprevotell

- g_Prevotella

g_Elusimicrobium

g_Lactobacillus

g_Clostridium s

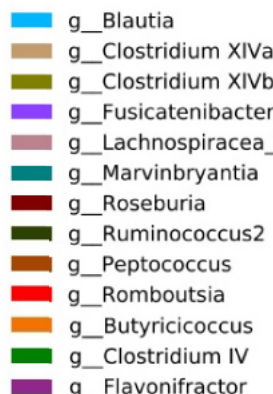

g_Intestinimonas

- g_Oscillibacter

g_Ruminococcus

g Turicibacter

- g_Phascolarctobacterium

- g_Parasutterella

g_Vampirovibrio

- g Helicobacter

g_Pseudomonas

- g_Anaeroplasm

g_Akkermansia

- g_other

FIGURE 2 | Bar plots of the gut microbiota compositions of each samples in 3 groups. (A) annotated at phylum level; (B) annotated at genus level. 

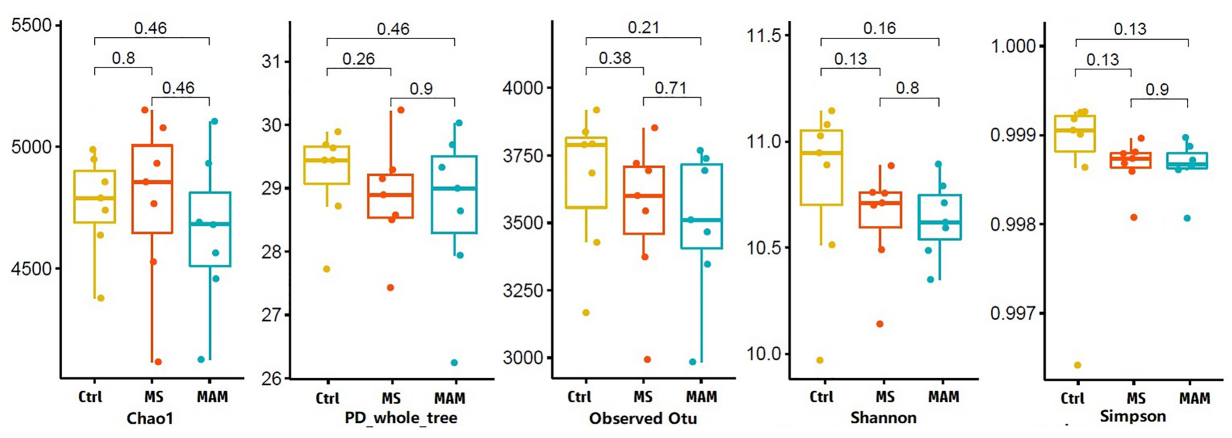

FIGURE 3 | Alpha diversity values for gut microbial communities among different model group rats.

(ko00730), methane metabolism (ko00680), and bacterial chemotaxis (ko02030).

\section{Mediation Analyses}

Mediation analyses were performed to investigate the possibility that gut microbiota composition mediates the association between early adversity and altered physiological variants. Among the 26 taxa correlated with MS exposure, Corynebacterium variation was found to potentially mediate the association of MS exposure and the visceral hypersensitivity variable corresponding to the distention threshold score of 2 for AWR. Among the 14 taxa identified at different taxonomic levels described above that correlated with MAM exposure, variable abundances of Butyricimonas and Butyricicoccus were found to potentially
A

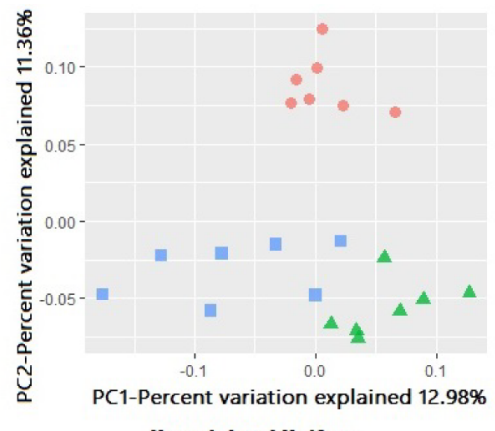

Unweighted Unifrac

C

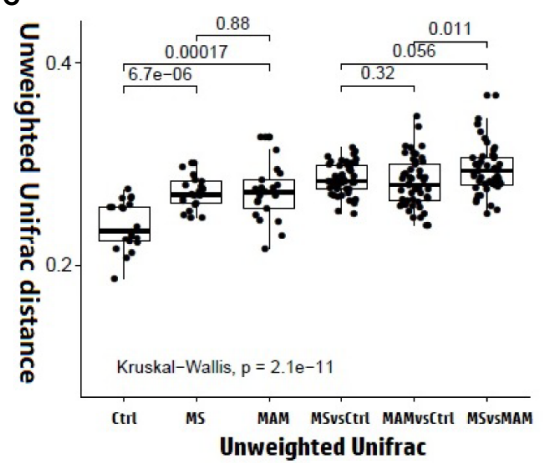

B
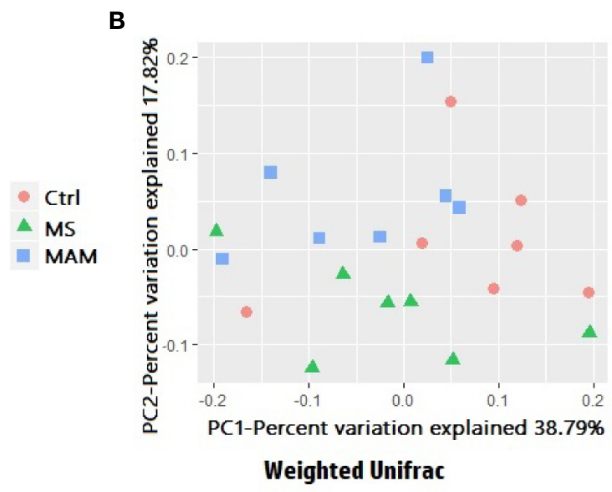

D

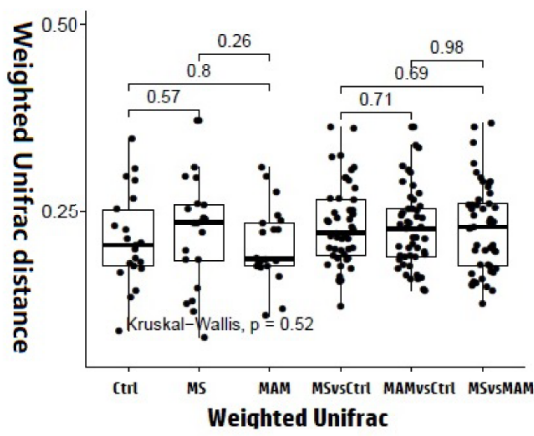

FIGURE 4 | Beta diversity analysis among and within different model groups. (A) 2D PCoA plots showing differences in the gut microbiota of rats from different model groups based on the unweighted Unifrac distance metric. $p=0.001$ and $r^{2}=0.21127$ in the Adonis test, $p=0.001$ and adjusted $r^{2}=0.123634$ in db-RDA analysis. (B) 2D PCoA plots showing differences in the gut microbiota of rats from different model groups based on the weighted Unifrac distance metric. $p=0.047$ and $r^{2}=0.1743$ in the Adonis test. $p=0.048$ and adjusted $r^{2}=0.069004$ in db-RDA analysis. (C) Comparison of inter-individual variation between and within different model groups based on the unweighted Unifrac distance metric. (D) Comparison of inter-individual variation between and within different model groups based on the weighted Unifrac distance metric. 
A

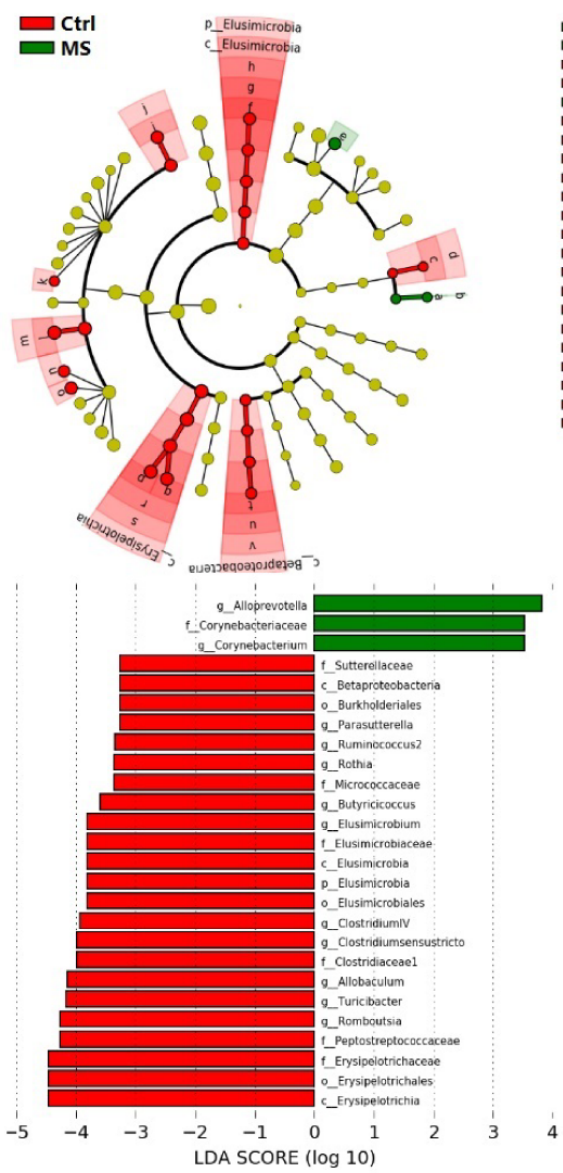

C

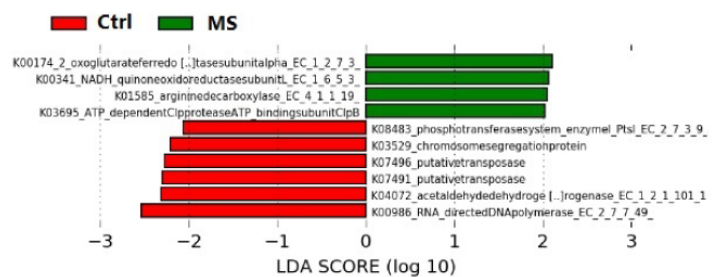

E

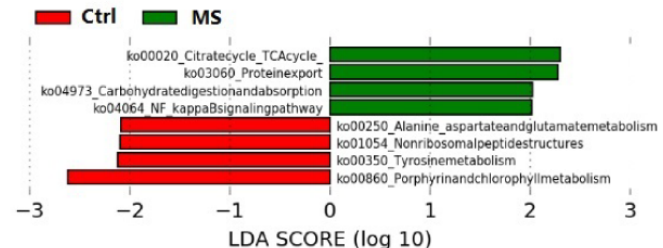

B
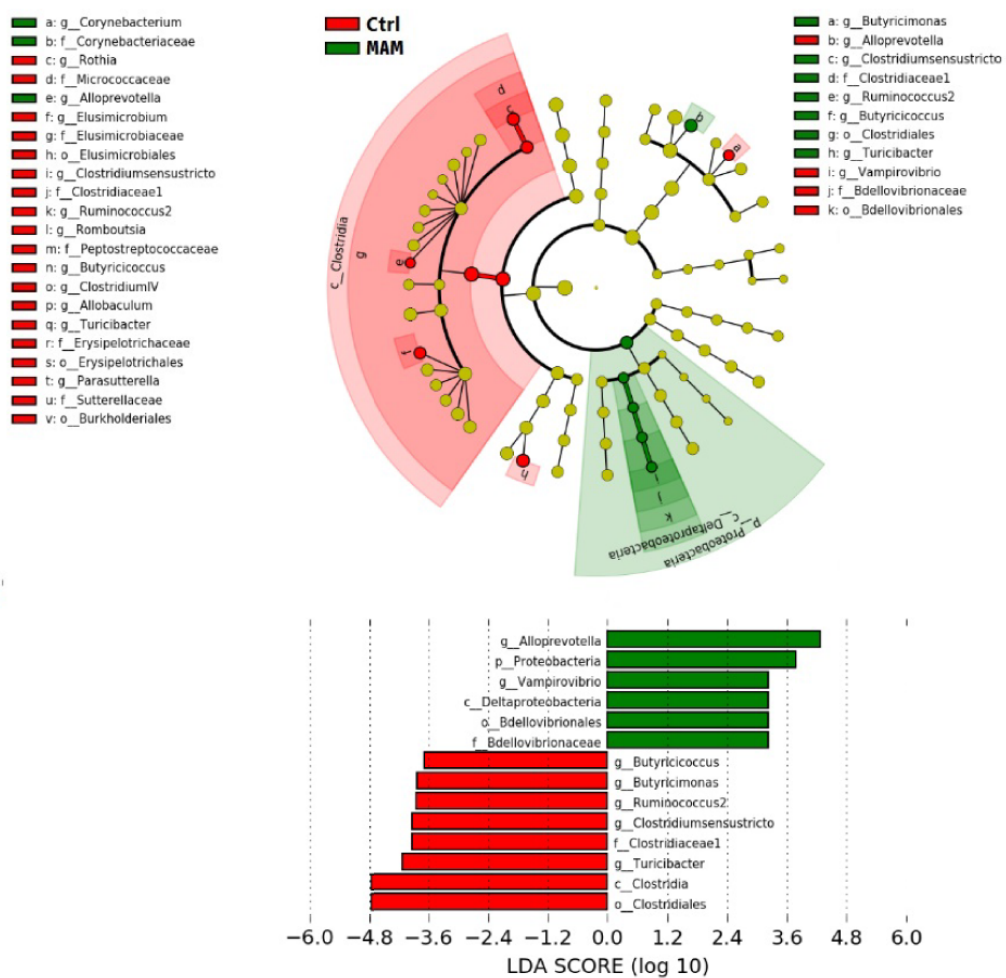

D
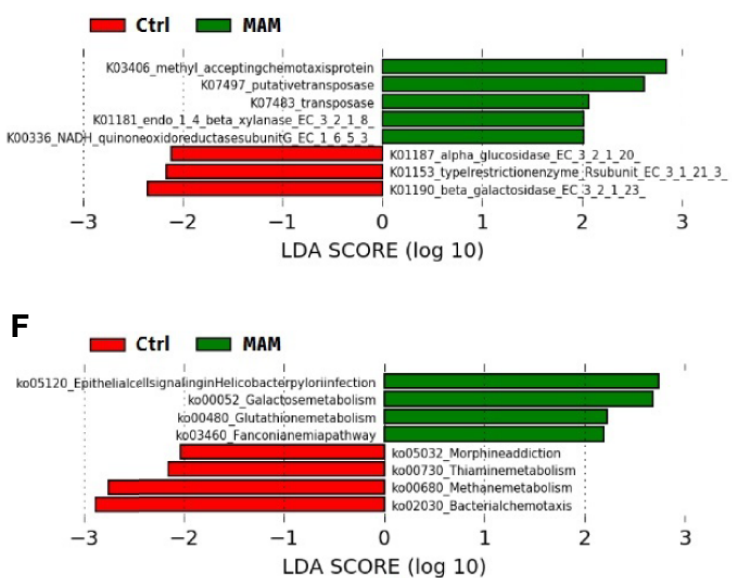

FIGURE 5 | Linear discriminant effect size (LEfSe) analyses comparing differentially abundant taxa and the KEGG-based ortholog functions and pathways between control and MS group communities and control and MAM group communities. (A, B) The differences in gut microbiota taxa between different model groups. The phylogenic relationship of the taxon with differences were shown in the cladogram. (C, D) Differences of KEGG-based ortholog (KO) inferred functions in gut microbiota between different model groups. (E, F) Differences in KO pathways predicted from gut microbiota compositions between different model groups. 
mediate MAM exposure-induced abnormalities in visceral hypersensitivity (Table 1).

\section{DISCUSSION}

In the present study, we established two IBS rat models using maternal separation (MS) and multiple early adversities (MAM) modeling and investigated their intestinal microbiota. Rats from both IBS models exhibited visceral hypersensitivity compared with the control group rats. However, the two model groups exhibited differences in the extent of visceral pain sensitivity and fecal water content. The microbial community structure from rats with the two visceral hypersensitivity models exhibited significant differences compared to the controls, while communities from the two model groups also exhibited significant differences between them. Furthermore, functional predictions of the gut microbiota suggested that rats from the two models exhibited different abundances of metabolisms and pathways associated with microbiota structural changes. Several distinct and common characteristic differences were also observed between rats from the two model groups. Some of these bacterial taxa may play a mediating role in the association between early adversity and specific pathological changes of IBS.

Several studies have evaluated early adversity events and alterations in intestinal microbiota diversity. Some of these have suggested that exposure to early adversity can affect the gut microbiota, manifesting as a reduction in microbial diversity and richness (O’Mahony et al., 2009; Qian et al., 2019). However, other studies have reported no changes in diversity in these scenarios (Moya-Perez et al., 2017). Comparison of five alphadiversity indices between different model groups did not yield any significant differences in this study. The apparent discrepancies in associations between microbial community alpha-diversity and early adversity could be explained by differences in study designs and environments, or even by factors of the same study including the type of early adverse events that were used and the timing of material collection, which can lead to variable results. For example, Zhou et al. (2016) evaluated the composition and diversity of the gut microbiota of MS, PI-IBS, and control rats at three different time points. No differences in alpha diversity were observed among the three groups in the third week and the eighth week, only the MS group exhibited a significant decrease in the Simpson and Shannon indices at week 12, while the PI-IBS group did not exhibit significant differences.

Inter-individual variation within host cohorts can also reflect the stability of gut microbiota. Healthy and homeostatic gut microbiota share higher similarities in the composition and richness of microbial taxa, while those with imbalanced microbiota exhibit drastic changes and tend to have higher inter-individual variation. Inter-group distances in the two model cohorts of this study exhibited significantly higher variation than those of the control group (Figure 4C). Thus, induced early negative events resulted in a significant increase in the instability of gut microbiota. exposure to early adversity can induce significant dysbiosis of fecal microbiota (Rincel et al., 2019).

To further evaluate the effects of different modeling processes on gut microbiota compositions, the composition and functionality differences of the communities among the model and control groups were further investigated. The two model groups exhibited several significantly altered taxonomic group abundances compared to the control group.

Bacterial taxonomic differences unique to the MS group involved 20 taxa related to 7 genera, namely, Corynebacterium, Rothia, Elusimicrobium, Romboutsia, Clostridium IV, Allobaculum and Parasutterella. Our finding that the abundances of Parasutterella species and their related taxonomic groups were significantly lower in the MS group compared to the control group was contrast with the result of Chen et al. (2018). The latter group found that Parasutterella was abundant in the stool of IBS patients. However, our results are consistent with other studies indicating that Parasutterella abundances are associated with beneficial outcomes. Zhang et al. (2019) reported that Parasutterella abundances were significantly lower in CDI patients and

TABLE 1 | Gut microbiota composition mediates the association between early adversity and altered physiological variants.

MAM exposure correlated alteration

\begin{tabular}{|c|c|c|c|c|c|c|c|}
\hline Taxa & Physiological Variants & \multicolumn{2}{|c|}{ ACME } & \multicolumn{2}{|c|}{ Total } & \multicolumn{2}{|c|}{ Prop. Mediated } \\
\hline Butyricimonas & AWR score at $50 \mathrm{mmHg}$ & 1.3 & 0.150 & 2.46 & $<0.001$ & 52.44 & 0.030 \\
\hline \multicolumn{8}{|c|}{ MS exposure correlated alteration } \\
\hline Taxa & Physiological Variants & \multicolumn{2}{|c|}{ ACME } & \multicolumn{2}{|c|}{ Total } & \multicolumn{2}{|c|}{ Prop. Mediated } \\
\hline Corynebacterium & Distention Threshold for AWR score 2 & -8.27 & 0.026 & -10 & 0.018 & 80.79 & 0.044 \\
\hline
\end{tabular}

ACME, average causal mediation effect; Prop. Mediated, proportion of mediation effect. 
asymptomatic carriers than in healthy controls, while Kreutzer et al. (2017) also reported a negative correlation between Parasutterella abundances and high-fat diet-induced hypothalamic inflammation. Although the association of Parasutterella and different health outcomes is clearly controversial, these observations nevertheless indicate that Parasutterella has a role in modulating the microbial activities and host responses in certain disease states and, thus, necessitate further investigation. The associations identified here between Corynebacterium, Rothia, Elusimicrobium, Romboutsia, Clostridium IV, and Allobaculum with maternal separation stress have not been reported elsewhere. Consequently, their associations with IBS require additional confirmation in further studies.

The bacterial taxonomic differences unique to the MAM group comprised five taxa, where the genus Butyricimonas decreased significantly and Vampirovibrio along with the family, order, class, and the phylum to which it belongs all increased significantly compared to the control group. This results were consistent with the previous studies suggested an association and role for genera Butyricimonas and Vampirovibrio with intestinal function (Yang et al., 2017; Song et al., 2020). Butyricimonas have been reported to produce butyrate that reduces inflammation and helps maintain healthy gut functioning (Yang et al., 2017). Vampirovibrio has been reported as significantly more abundant in trinitrobenzene sulfonic acid-induced PI-IBS rat model individuals, and its lowered abundance has been associated with the alleviation of symptoms for visceral hypersensitivity after treatment (Song et al., 2020).

Predictive analysis of microbiota functionalities also suggested that changes in the microbial composition of the two model groups corresponded to functional differences associated with diverse metabolisms and pathways.

Specifically, we observed significant different abundances of the 8 pathways in the MS group compared to the control group. Among them, the enhanced functional capacity for the NFkappa B signaling pathway in MS group is consistent with previous studies indicating that neonatal MS induces $\mathrm{VH}$ and visceral pain in rats that is mediated by activation of TLR4 and the NF- $\mathrm{KB}$ signaling pathway (Chen et al., 2015; Yuan et al., 2015) and further suggests that changes in the gut microbiomes caused by MS are involved in NF- $\mathrm{KB}$ activation in a rat model of IBS.

In the MAM group, we also found 8 significantly different pathways compared to the control group, which is distinct from that of MS group, suggest that different modeling strategies may result in IBS symptoms by inducing changes in microbiota via different metabolic pathways.

Despite the significant differences in microbial composition and functionalities among the two model groups, six taxa were common in both the model groups relative to the control group communities. Specifically, Alloprevotella were more abundant in both the MS and MAM groups, while Butyricicoccus, Turicibacter, Ruminococcus, and Clostridium sensu stricto along with the family it belongs to were less abundant in both the MS and MAM groups relative to controls. These results were consistent with those from previous studies. For example, Alloprevotella were significantly more abundant in model rats with early adversity stimuli compared to controls in one study (Rincel et al., 2019). In addition, Turicibacter were significantly less abundant in IBS-D patients (Zhuang et al., 2018). Ruminococcus, Butyricicoccus, and Clostridium sensu stricto are all short-chain fatty acid-producing genera that are considered beneficial for gastrointestinal tract functioning (Eeckhaut et al., 2013; Kong et al., 2019; Perez-Burillo et al., 2019). Furthermore, Ruminococcus spp. were less abundant in IBS patients relative to controls in another study (Mack et al., 2019). Moreover, several studies have shown that the abundances of Butyricicoccus were lower in ulcerative colitis patients and patients with inflammatory disease in general (Eeckhaut et al., 2013; Rios-Covian et al., 2016; Devriese et al., 2017). These microbial signatures exhibited similar abundance patterns in both early adversity modeling procedures, indicating that the responsive species may be involved in common characteristics of the complex pathophysiological changes that cause IBS. Thus, the variation in these taxa could provide clues to the etiology of IBS and potentially lead to novel therapies.

The changes in the gut microbiota and pathophysiological indicators described in this study were both induced by early adversity events. To further assess the contribution of altered gut microbial compositions to the association between early adversity and altered physiological outcomes, mediation analysis was performed to investigate the potential mediating effects of microbial populations on pathophysiological changes induced by early adversity events. Corynebacterium was predicted to partially mediate MS exposure-induced visceral hypersensitivity, while Butyricimonas and Butyricicoccus populations were predicted to partially mediate MAM exposure-induced visceral hypersensitivity. Thus, these genera might play critical roles in the development of early adversityinduced visceral hypersensitivity and may also represent targets for understanding the mechanisms underlying microbemediated visceral hypersensitivity.

A limitation of the present study is the lack of proven causal relationships between alterations in microbiota characteristics and visceral pain behaviors, despite the abundance of evidence suggesting that microbial compositions play roles in the development of visceral hypersensitivity. Future studies could deconvolute these effects by focusing on manipulating the gut microbiota through prebiotics, probiotics, or synbiotics to reverse the deleterious effects of early adversarial events on visceral pain or other IBS-related behaviors.

To conclude, the present study demonstrated that MS stress and multiple early adversity stress procedures could induce distinct alterations of the gut microbiota that also differ in stability, composition, and functionality in rat models. The large differences in gut microbiota between the two model groups coincide with the heterogeneity and complexity of IBS pathogenesis and highlight the importance in evaluating gut microbiota characteristics in IBS research while also systematically considering potential modeling procedural differences. The 
microbial compositional and functional differences identified in this study prompt further investigation of mechanisms of early adversity induced IBS related pathophysiological changes.

\section{DATA AVAILABILITY STATEMENT}

The datasets presented in this study can be found in online repositories. The names of the repository/repositories and accession number(s) can be found below: https://www.ncbi. nlm.nih.gov/, PRJNA609270.

\section{ETHICS STATEMENT}

The animal study was reviewed and approved by the Ethics Committee of Minzu University of China.

\section{AUTHOR CONTRIBUTIONS}

WE, SJ, and LY performed the experiments. WE and SJ analyzed the data. PL and LY obtained funding for the project and planned

\section{REFERENCES}

Aroniadis, O. C., Brandt, L. J., Oneto, C., Feuerstadt, P., Sherman, A., Wolkoff, A. W., et al. (2019). Faecal microbiota transplantation for diarrhoea-predominant irritable bowel syndrome: a double-blind, randomised, placebo-controlled trial. Lancet Gastroenterol. Hepatol. 4, 675-685. doi: 10.1016/S2468-1253(19)30198-0

Chen, Z. Y., Zhang, X. W., Yu, L., Hua, R., Zhao, X. P., Qin, X., et al. (2015). Spinal toll-like receptor 4-mediated signalling pathway contributes to visceral hypersensitivity induced by neonatal colonic irritation in rats. Eur. J. Pain 19, 176-186. doi: 10.1002/ejp.534

Chen, Y. J., Wu, H., Wu, S. D., Lu, N., Wang, Y. T., Liu, H. N., et al. (2018). Parasutterella, in association with irritable bowel syndrome and intestinal chronic inflammation. J. Gastroenterol. Hepatol. 33, 1844-1852. doi: 10.1111/jgh.14281

Devriese, S., Eeckhaut, V., Geirnaert, A., Van den Bossche, L., Hindryckx, P., Van de Wiele, T., et al. (2017). Reduced Mucosa-associated Butyricicoccus Activity in Patients with Ulcerative Colitis Correlates with Aberrant Claudin-1 Expression. J. Crohns Colitis 11, 229-236. doi: 10.1093/ecco-jcc/jjw142

Eeckhaut, V., Machiels, K., Perrier, C., Romero, C., Maes, S., Flahou, B., et al. (2013). Butyricicoccus pullicaecorum in inflammatory bowel disease. Gut 62, 1745-1752. doi: 10.1136/gutjnl-2012-303611

Ford, A. C., Lacy, B. E., and Talley, N. J. (2017). Irritable Bowel Syndrome. N. Engl. J. Med. 376, 2566-2578. doi: 10.1056/NEJMra1607547

Fukui, H., Oshima, T., Tanaka, Y., Oikawa, Y., Makizaki, Y., Ohno, H., et al. (2018). Effect of probiotic Bifidobacterium bifidum G9-1 on the relationship between gut microbiota profile and stress sensitivity in maternally separated rats. Sci. Rep. 8, 12384. doi: 10.1038/s41598-018-30943-3

Gong, Z., Chen, Y., Zhang, R., Wang, Y., Yang, Q., Guo, Y., et al. (2014). Pharmacokinetics of two alkaloids after oral administration of rhizoma coptidis extract in normal rats and irritable bowel syndrome rats. Evid. Based Complement. Alternat. Med. 2014, 845048. doi: 10.1155/2014/845048

Halland, M., and Saito, Y. A. (2015). Irritable bowel syndrome: new and emerging treatments. BMJ 350, h1622. doi: 10.1136/bmj.h1622

Holtmann, G. J., Ford, A. C., and Talley, N. J. (2016). Pathophysiology of irritable bowel syndrome. Lancet Gastroenterol. Hepatol. 1, 133-146. doi: 10.1016/ S2468-1253(16)30023-1

Ju, T., Kong, J. Y., Stothard, P., and Willing, B. P. (2019). Defining the role of Parasutterella, a previously uncharacterized member of the core gut microbiota. ISME J. 13, 1520-1534. doi: 10.1038/s41396-019-0364-5 the experiments. WE, PL, and LY prepared the manuscript draft for submission. All authors contributed to the article and approved the submitted version.

\section{FUNDING}

The study of the rat's physiological parameters and the histopathological analysis was funded by the foundation of Key Laboratory of Ethnomedicine (Minzu University of China), Ministry of Education (KLEM-ZZ201903, KLEMZZ2020GD01) and Master's Independent Research Project of Minzu University of China (SSZZKY-20200027), the analysis of fecal microbiota was funded by the National Natural Science Foundation of China (81473451 and 81673769). The funding bodies had no role in the design of the study, collection, analysis, or interpretation of data or in writing the manuscript.

\section{ACKNOWLEDGMENTS}

This manuscript has been released as a pre-print at Research square.

Kong, C., Gao, R., Yan, X., Huang, L., and Qin, H. (2019). Probiotics improve gut microbiota dysbiosis in obese mice fed a high-fat or high-sucrose diet. Nutrition 60, 175-184. doi: 10.1016/j.nut.2018.10.002

Kreutzer, C., Peters, S., Schulte, D. M., Fangmann, D., Turk, K., Wolff, S., et al. (2017). Hypothalamic Inflammation in Human Obesity Is Mediated by Environmental and Genetic Factors. Diabetes 66, 2407-2415. doi: 10.2337/ db17-0067

Li, Y., Du, Y., Zhang, A., Jiang, R., Nie, X., and Xiong, X. (2019). Role of CCR7 on dendritic cellmediated immune tolerance in the airways of allergyinduced asthmatic rats. Mol. Med. Rep. 20, 4425-4432. doi: 10.3892/mmr.2019.10694

Ma, J., Li, J., Qian, M., He, N., Cao, Y., Liu, Y., et al. (2019). The comprehensive pathophysiological changes in a novel rat model of postinflammatory visceral hypersensitivity. FASEB J. 33 (12), 13560-13571. doi: 10.1096/fj.201901489R

Mack, A., Bobardt, J. S., Hass, A., Nichols, K. B., Schmid, R. M., and SteinThoeringer, C. K. (2019). Changes in gut microbial metagenomic pathways associated with clinical outcomes after the elimination of malabsorbed sugars in an IBS cohort. Gut Microbes 11 (3), 620-631. doi: 10.1080/ 19490976.2019.1686322

Moya-Perez, A., Perez-Villalba, A., Benitez-Paez, A., Campillo, I., and Sanz, Y. (2017). Bifidobacterium CECT 7765 modulates early stress-induced immune, neuroendocrine and behavioral alterations in mice. Brain Behav. Immun. 65, 43-56. doi: 10.1016/j.bbi.2017.05.011

O’Mahony, S. M., Marchesi, J. R., Scully, P., Codling, C., Ceolho, A. M., Quigley, E. M., et al. (2009). Early life stress alters behavior, immunity, and microbiota in rats: implications for irritable bowel syndrome and psychiatric illnesses. Biol. Psychiatry 65, 263-267. doi: 10.1016/j.biopsych.2008.06.026

Parasuraman, S., Raveendran, R., and Kesavan, R. (2010). Blood sample collection in small laboratory animals. J. Pharmacol. Pharmacother. 1, 87-93. doi: 10.4103/0976-500X.72350

Perez-Burillo, S., Pastoriza, S., Fernandez-Arteaga, A., Luzon, G., JimenezHernandez, N., D’Auria, G., et al. (2019). Spent Coffee Grounds Extract, Rich in Mannooligosaccharides, Promotes a Healthier Gut Microbial Community in a Dose-Dependent Manner. J. Agric. Food Chem. 67, 25002509. doi: 10.1021/acs.jafc.8b06604

Qian, L., Lu, L., Huang, L., Wen, Q., Xie, J., Jin, W., et al. (2019). The effect of neonatal maternal separation on short-chain fatty acids and airway inflammation in adult asthma mice. Allergol. Immunopathol. (Madr.) 47, $2-$ 11. doi: 10.1016/j.aller.2018.05.004 
Rincel, M., and Darnaudery, M. (2019). Maternal separation in rodents: a journey from gut to brain and nutritional perspectives. Proc. Nutr. Soc. 79 (1), 113-132. doi: 10.1017/S0029665119000958

Rincel, M., Aubert, P., Chevalier, J., Grohard, P. A., Basso, L., Monchaux de Oliveira, C., et al. (2019). Multi-hit early life adversity affects gut microbiota, brain and behavior in a sex-dependent manner. Brain Behav. Immun. 80, 179192. doi: 10.1016/j.bbi.2019.03.006

Rios-Covian, D., Ruas-Madiedo, P., Margolles, A., Gueimonde, M., de Los ReyesGavilan, C. G., and Salazar, N. (2016). Intestinal Short Chain Fatty Acids and their Link with Diet and Human Health. Front. Microbiol. 7, 185. doi: 10.3389/ fmicb.2016.00185

Song, Y. F., Pei, L. X., Chen, L., Geng, H., Yuan, M. Q., Xu, W. L., et al. (2020). Electroacupuncture Relieves Irritable Bowel Syndrome by Regulating IL-18 and Gut Microbial Dysbiosis in a Trinitrobenzene Sulfonic Acid-Induced PostInflammatory Animal Model. Am. J. Chin. Med. 48 (1), 77-90. doi: 10.1142/ S0192415X20500950

Tap, J., Derrien, M., Tornblom, H., Brazeilles, R., Cools-Portier, S., Dore, J., et al. (2017). Identification of an Intestinal Microbiota Signature Associated With Severity of Irritable Bowel Syndrome. Gastroenterology 152, 111-123.e8. doi: 10.1053/j.gastro.2016.09.049

Wang, H. J., Xu, X., Xie, R. H., Rui, Y. Y., Zhang, P. A., Zhu, X. J., et al. (2018). Prenatal maternal stress induces visceral hypersensitivity of adult rat offspring through activation of cystathionine-beta-synthase signaling in primary sensory neurons. Mol. Pain 14, 1744806918777406. doi: 10.1177/ 1744806918777406

Xu, G. Y., Winston, J. H., Shenoy, M., Zhou, S., Chen, J. D., and Pasricha, P. J. (2009). The endogenous hydrogen sulfide producing enzyme cystathioninebeta synthase contributes to visceral hypersensitivity in a rat model of irritable bowel syndrome. Mol. Pain 5, 44. doi: 10.1186/1744-8069-5-44

Yang, C., Qu, Y., Fujita, Y., Ren, Q., Ma, M., Dong, C., et al. (2017). Possible role of the gut microbiota-brain axis in the antidepressant effects of (R)-ketamine in a social defeat stress model. Transl. Psychiatry 7, 1294. doi: 10.1038/s41398-0170031-4

Yuan, B., Tang, W. H., Lu, L. J., Zhou, Y., Zhu, H. Y., Zhou, Y. L., et al. (2015). TLR4 upregulates CBS expression through NF-kappaB activation in a rat model of irritable bowel syndrome with chronic visceral hypersensitivity. World J. Gastroenterol. 21, 8615-8628. doi: 10.3748/wjg.v21.i28.8615

Zhang, J., Song, L., Wang, Y., Liu, C., Zhang, L., Zhu, S., et al. (2019). Beneficial effect of butyrate-producing Lachnospiraceae on stress-induced visceral hypersensitivity in rats. J. Gastroenterol. Hepatol. 34, 1368-1376. doi: 10.1111/jgh.14536

Zhou, X. Y., Li, M., Li, X., Long, X., Zuo, X. L., Hou, X. H., et al. (2016). Visceral hypersensitive rats share common dysbiosis features with irritable bowel syndrome patients. World J. Gastroenterol. 22, 5211-5227. doi: 10.3748/wjg.v22.i22.5211

Zhuang, Z., Zhang, L., Wang, X., Tao, L., and Lv, B. (2016). PDIA3 gene induces visceral hypersensitivity in rats with irritable bowel syndrome through the dendritic cell-mediated activation of T cells. PeerJ 4, e2644. doi: 10.7717/ peerj. 2644

Zhuang, X., Tian, Z., Li, L., Zeng, Z., Chen, M., and Xiong, L. (2018). Fecal Microbiota Alterations Associated With Diarrhea-Predominant Irritable Bowel Syndrome. Front. Microbiol. 9, 1600. doi: 10.3389/fmicb.2018.01600

Conflict of Interest: The authors declare that the research was conducted in the absence of any commercial or financial relationships that could be construed as a potential conflict of interest.

Copyright (C) 2021 Enqi, Jingzhu, Lingpeng and Yaqin. This is an open-access article distributed under the terms of the Creative Commons Attribution License (CC BY). The use, distribution or reproduction in other forums is permitted, provided the original author(s) and the copyright owner(s) are credited and that the original publication in this journal is cited, in accordance with accepted academic practice. No use, distribution or reproduction is permitted which does not comply with these terms. 